Original Article

\title{
Effect of Glucosamine Sulfate Flone and Combined with Moderate Intensity Exercise on Serum Levels of CS 846 Epitope and Cartilage Oligomeric Matrix Protein in a Rat Model of Osteoarthritis
}

Babak Biniaz

(PhD Candidate) Department of Sport Physiology, Sari Branch, Islamic Azad University, Sari, Iran

Hajar Abbaszadeh î

(PhD) Department of Sport Physiology, Sari Branch, Islamic Azad University, Sari, Iran

Parvin Farzanegi iD

(PhD) Department of Sport Physiology, Sari Branch, Islamic Azad University, Sari, Iran

Corresponding author: Hajar Abbaszadeh

Tel: +989112163511

Email:h.abaszade61@gmail.com

Address: Department of Sport

Physiology, Sari Branch, Islamic Azad

University, Sari, Iran

Received: 2019/11/24

Revised: $2020 / 01 / 20$

Accepted: 2020/01/27

\section{(c) $(1) \Theta$}

This work is licensed under a Creative

Commons Attribution 4.0 License.

DOI: $10.29252 / \mathrm{mlj} .15 .2 .35$

\section{ABSTRACT}

Background and objectives: 0steoarthritis is the result of a defect in synovial membrane-covered joint tissues. The purpose of this study was to investigate effects of glucosamine sulfate alone and combined with moderate intensity exercise on serum levels of CS 846 epitope and cartilage oligomeric matrix protein (COMP) in a rat model of osteoarthritis.

Methods: In this study, after inducing osteoarthritis in 42 male Wistar rats (weighting $250 \pm 300 \mathrm{~g}$, aged 8 to 12 weeks), the rats were randomly divided into five groups: control-healthy, control-patient, patient-exercise, patient-glucosamine and patientglucosamine-exercise. The training program consisted of 30 minutes of running on a non-slip treadmill at speed of $16 \mathrm{~m} / \mathrm{min}$ in the first week with progressive overload principle reaching 50 minutes by the eighth week. The glucosamine groups received oral glucosamine sulfate (250 mg/kg/day) once a day for eight consecutive weeks. The serum levels of CS 846 epitope and CONP were measured using commercial ELISA kits. Data were analyzed using one-way ANOVA and Tukey's post hoc test. All statistical analyses were performed in GraghPad prism 8 and at significance level of 0.05 .

Results: Combined exercise and glucosamine supplementation caused a significant decrease in the COMP and CS846 levels $(\mathrm{P}<0.0001)$. This decrease was more profound compared to that of glucosamine and exercise alone.

Conclusion: 0verall, the findings of the present study showed that osteoarthritis increases serum COMP and CS 846 levels. In addition, glucosamine supplementation combined with exercise can significantly improve knee osteoarthritis in rats.

KEYWORDS: Cartilage oligomeric matrix protein, epitopes, Exercise, Glucosamine, 0steoarthritis 


\section{INTRODUCTION}

Osteoarthritis (OA) is the result of a defect in a synovial membrane-covered joint (1). The prevalence of OA in Iran is about 16\% in individuals aged 15-82 years (2). The symptomatic prevalence of this disease is $10 \%$ in men and $13 \%$ in women over 65 years in the United States (3). Clinical symptoms include pain, joint stiffness, joint effusion, progressive muscle weakness and atrophy, instability, impaired articulation as well as contraction and movement limitations, which affect the patients' ability to perform activities that require weight-bearing (4). Undoubtedly, the discovery of biochemical markers for early detection of OA will be useful in the development of new drug therapies aimed at improving OA before its irreversibility (5). Concentrations of specific cartilage and bone molecules that reflect tissue remodeling in OA patients are considered as prognostic factors for the extent of joint destruction. Compared to normal population, an increase in cartilage oligomeric matrix protein (COMP) concentration was found in all patients with rapid knee arthroplasty. In contrast, the level of a well-known marker of cumulative cartilage synthesis, called chondroitin sulphate epitope 846 (CS846) is elevated only in patients with slow articular destruction. Therefore, high serum levels of COMP may indicate an unfavorable prognosis for rapid destruction of the joint, while an increase in the CS846 indicates a favorable prognosis (6). COMP is a prominent component of cartilage matrix. This glycoprotein plays an important role in regulating the assembly (production) of collagen type II fibers in cartilage and, in cooperation with other matrix proteins, stabilizes the collagen network. Serum COMP concentration is increased in patients with knee OA. In addition, studies show that elevated serum COMP levels are associated with progressive articular injury in knee OA (7). CS846 is also found on newly synthesized aggrecan fragments that are released from the cartilage matrix and can act as a marker of cartilage metabolism (8).

Numerous pharmacological, surgical and nonpharmacological treatments have been proposed for OA $(9,10)$. In recent years, glucosamine supplements have received much attention in the treatment of $\mathrm{OA}$ and other similar diseases $(11,12)$. Glucosamine is an amino monosaccharide synthesized from glucose that is converted to glycosaminoglycan (5). Glucosamine is a major component of proteoglycans and is found naturally in articular cartilage and synovial fluid (13). It has been reported that oral glucosamine can reduce pain and improve mobility in OA patients with no notable side effects $(5,14,15)$. Mohsin et al. reported that $500 \mathrm{mg}$ oral glucoseamine three times a day for six weeks improve pain, swelling and mobility in OA patients (16). Another study also indicated that both topical and oral glucosamine have positive effects on symptoms and pain in OA patients (17).

$\mathrm{OA}$ is often treated with nonsteroidal antiinflammatory drugs (NSAIDs) or glucosamine. These treatments may relieve pain, but their effect on cartilage and synovium metabolism in patients with $\mathrm{OA}$ is controversial. In vitro studies have shown that NSAIDs may lower cartilage proteoglycan synthesis (7). There are limited clinical evidence on the effects of nonpharmacological interventions for treating and controlling OA symptoms (18). It is welldemonstrated that exercise reduces pain and improves performance in people with knee OA (7). Some studies indicated that high-intensity exercise can cause pain in patients with osteoarthritis (19), while moderate-intensity exercise have positive effects $(20,21)$. It has been shown that an acute period of exercise could increase serum COMP in healthy adults as well as in patients with knee OA, whereas six weeks of chronic exercise has positive effects on COMP blood levels (7). A limited number of studies has examined the effect of NSAIDs on serum COMP levels in patients with OA along with exercise training (22). The present study aimed to evaluate effects of glucosamine supplementation on COPM and CS846 levels in cartilage tissue of OA rats exposed to weight-bearing exercise.

\section{MATERIAL AND METHODS}

This experimental study was performed on 42 male Wistar rats aged 8-12 weeks with a mean initial weight of 250 to $300 \mathrm{~g}$. The rats were obtained from the research center of Islamic Azad University, Sari Branch and were divided into five groups: control-healthy, control-patient, patient-exercise, patientglucosamine and patient-glucosamineexercise. The study was approved by the 
animal care and use committee at the Islamic Azad University of Sari (approval number: IR.IAU.SARI.REC.1398.132). The rats were housed in a room with controlled conditions (at ambient temperature of $20 \pm 2{ }^{\circ} \mathrm{C}$, humidity of $50 \% \pm 5$ and 12:12 light dark cycle). The animals were also fed $10 \mathrm{~g} / 100 \mathrm{~g}$ body weight daily (according to weekly weight gain). Induction of $\mathrm{OA}$ by surgical procedure was carried out based on the method described by Malfait and Little (23). The rats were anesthetized with ketamine $(30-50 \mathrm{mg} / \mathrm{kg})$ and xylocaine $(3-5 \mathrm{mg} / \mathrm{kg})$. Then, $1 \mathrm{~cm}$ incision was made from the right knee to expose knee joint. The knee joint was opened by lateral displacement of the patella and its ligament, and a longitudinal incision was made in the medial part of the knee. The lateral displacement of the patella and patellar ligament was performed by forceps and then an incomplete incision was made in the anterior cruciate ligament without damaging the articular cartilage and other ligaments. Finally, articular capsule and skin were closed with six absorbable sutures (23). One month after the surgery, the rats were subjected to 10minute treadmill running, three days a week at a speed of $16 \mathrm{~m} / \mathrm{min}, 60-70 \% \mathrm{VO}_{2} \max$ and zero slope. No stimulant was used to move the rats. Details of the training program are presented in table 1 .

The glucosamine group received oral glucosamine sulfate $(250 \mathrm{mg} / \mathrm{kg} /$ day $)$ once a day for eight consecutive weeks, through gavage (24). The glucoseamine+exercise group received this dose before each exercise session. To evaluate the recovery rate in OA, observable motor behaviors including walking and weight bearing in the injured leg were observed. After 12 to 14 hours of fasting and 48 hours after the interventions (to eliminate the acute effects of exercise and oral glucosamine), the animals were first anesthetized with intraperitoneal injections of ketamine $(60 \mathrm{mg} / \mathrm{kg})$ and xylocaine $(5 \mathrm{mg} / \mathrm{kg})$ and then sacrificed. After the abdominal cavity was opened, blood samples were taken and frozen at $-80{ }^{\circ} \mathrm{C}$ for analysis. Level of COPM and epitope CS846 was measured using enzyme-linked immunosorbent assay (ELISA) kits according to manufacturer's instructions (IBEX Montreal, Quebec, Canada). Data were expressed as mean \pm standard deviation (SD). Data analysis was performed in GraghPad prism 8 software using the Shapiro-Wilk's test, Leven's test, one-way ANOVA and Tukey's post hoc test. All statistical analyses were performed at significance level of 0.05 .

Table 1- Details of the training protocol

\begin{tabular}{|c|c|c|c|c|c|c|c|c|c|}
\hline Variable & Compatibility & $1^{\text {st }}$. week & $\begin{array}{l}2^{\text {nd }} . \\
\text { week }\end{array}$ & $\begin{array}{l}3^{\text {rd }} \cdot \\
\text { week }\end{array}$ & $4^{\text {th }}$. week & $\begin{array}{l}5^{\text {th }} . \\
\text { week }\end{array}$ & $\begin{array}{l}6^{\text {th }} . \\
\text { week }\end{array}$ & $\begin{array}{l}7^{\text {th }} . \\
\text { week }\end{array}$ & $\begin{array}{l}8^{\text {th. }} . \\
\text { week }\end{array}$ \\
\hline $\begin{array}{ll}\text { Turning } & \text { Speed } \\
(\mathrm{m} / \mathrm{min}) & \end{array}$ & 16 & 16 & 16 & 16 & 16 & 16 & 16 & 16 & 16 \\
\hline $\begin{array}{l}\text { Practice time per } \\
\text { session (minutes) }\end{array}$ & 10 & 30 & 30 & 30 & 50 & 50 & 50 & 50 & 50 \\
\hline
\end{tabular}

\section{RESULTS}

Results of ANOVA showed a significant difference in COMP and CS846 levels between the groups (Table 2). The combination of exercise and glucosamine supplementation caused a significant increase in the mean COMP and CS846 levels compared with the normal group, and a significant decrease compared with the patient and patient glucosamine groups (Table 3). COMP level in the patient+glucosamine group and the patient+exercise group was significantly lower than in the patient group. CS846 level in the patient+exercise group was significantly lower than in the patient group (Table 3). More importantly, the decrease in the COMP and CS846 levels after combined exercise and glucosamine supplementation was more profound compared to that after glucosamine and exercise alone $(\mathrm{P}<0.0001)$. COMP and CS846 levels did not differ significantly between the exercise+glucosamine group and the exercise group (Table 3). Moreover, CS846 level did not differ significantly between the patient group and the patient+ glucosamine group (Table 3). 
Table 2- Differences in the level of COMP and CS846 between the study groups based on one-way ANOVA

\begin{tabular}{|c|c|c|c|c|c|c|}
\hline Variable & ANOVA table & SS & DF & MS & $\mathbf{F}$ & $\mathbf{P}$ \\
\hline \multirow[t]{3}{*}{$\operatorname{COMP}(\mu \mathrm{g} / \mathrm{l})$} & Treatment (Intergroup) & 21.50 & 4 & 5.375 & 89.21 & $\mathbf{P}<0.0001$ \\
\hline & Residual (Intragroup) & 1.205 & 20 & 0.06026 & & \\
\hline & Total & 22.71 & 24 & & & \\
\hline \multirow[t]{3}{*}{ CS846 (ng/l) } & Treatment (Intergroup) & 17.55 & 4 & 4.387 & 64.79 & $P<0.0001$ \\
\hline & Residual (Intragroup) & 1.354 & 20 & 0.06771 & & \\
\hline & Total & 18.90 & 24 & & & \\
\hline
\end{tabular}

Table 3- Comparison of COMP and CS846 levels between the study groups based on the Tukey's multiple comparison test

\begin{tabular}{|c|c|c|c|c|c|c|}
\hline & $\begin{array}{l}\text { Mean 1 } \\
\text { (COMP) }\end{array}$ & $\begin{array}{c}\text { Mean } 2 \\
\text { (COMP) }\end{array}$ & $\begin{array}{l}\text { P-Value } \\
\text { (COMP) }\end{array}$ & $\begin{array}{l}\text { Mean } 1 \\
(\text { CS846) }\end{array}$ & $\begin{array}{l}\text { Mean } 2 \\
(\text { CS846) }\end{array}$ & $\begin{array}{l}\text { P-Value } \\
\text { (CS846) }\end{array}$ \\
\hline Normal vs. Patient & 1.052 & 3.842 & $<0.0001$ & 1.624 & 4.050 & $<0.0001$ \\
\hline Normal vs. Patient+Glu & 1.052 & 2.962 & $<0.0001$ & 1.624 & 3.740 & $<0.0001$ \\
\hline Normal vs. Patient+Exe & 1.052 & 2.510 & $<0.0001$ & 1.624 & 3.006 & $<0.0001$ \\
\hline Normal vs. Patient+Exe+Glu & 1.052 & 2.064 & $<0.0001$ & 1.624 & 2.994 & $<0.0001$ \\
\hline Patient vs. Patient+Glu & 3.842 & 2.962 & 0.0001 & 4.050 & 3.740 & 0.3573 \\
\hline Patient vs. Patient+Exe & 3.842 & 2.510 & $<0.0001$ & 4.050 & 3.006 & $<0.0001$ \\
\hline Patient vs. Patient+Exe+Glu & 3.842 & 2.064 & $<0.0001$ & 4.050 & 2.994 & $<0.0001$ \\
\hline Patient+Glu vs. Patient+Exe & 2.962 & 2.510 & 0.0589 & 3.740 & 3.006 & 0.0020 \\
\hline Patient+Glu vs. Patient+Exe+Glu & 2.962 & 2.064 & 0.0001 & 3.740 & 2.994 & 0.0017 \\
\hline Patient+Exe vs. Patient+Exe+Glu & 2.510 & 2.064 & 0.0637 & 3.006 & 2.994 & $>0.9999$ \\
\hline
\end{tabular}

\section{DISCUSSION}

$\mathrm{OA}$ is characterized by progressive destruction of articular cartilage associated with subchondral bone regeneration, marginal osteophyte formation and loss of progressive symptoms of mechanical function (25). Early destruction of articular cartilage in OA begins with the loss of glycosaminoglycan (GAG) from the articular cartilage surface followed by collagenolysis. Consequently, early markers of articular cartilage injury or OA changes may be found among GAG metabolic products (5). Thus, we measured cartilage tissue degradation in OA rats by measuring COMP and CS846 levels. The results showed that levels of COMP and CS 846 abnormally increased in the OA rat models. CS is a GAG that is covalently attached to specific proteins to form proteoglycans. The level of aggrecan synthesis can be assessed using antibodies against CS846, which is located in the chondroitin sulfate chain. In contrast, the ratio of CS846 to aggrecan was high in the more advanced stages of OA, indicating that both the synthesis and degradation of aggrecan were up-regulated. The correlation between the histology of cartilage injury and the change in CS846 concentration indicates that aggrecan synthesis in severely damaged cartilage is increased (5). COMP is also a non-collagenous extracellular matrix glycoprotein that contains domains such as thrombospondin that can bind to different types of collagen and is responsible for collagen-collagen interactions and the formation of micro-fibrils in cartilage. Increased serum COMP concentration has been reported in several cohort studies on OA samples and is thought to be associated with OA severity. Recent evidence suggests that a high concentration of COMP can be detected in the affected joints years after injury or in the serum of patients with OA after exercise, indicating that COMP is a very sensitive marker for early asymptomatic OA. However, some studies indicate that serum COMP levels cannot be a reliable diagnostic marker for primary OA. One reason for this may be that in early OA, cartilage damage is not significant yet; therefore, the serum COMP level may be influenced by the turnover of other types of cartilage in the body (for example, rib cartilage) rather than damage (if any) to the cartilage in the primary OA joint (25). A previous study demonstrated a relationship between serum COMP level and CS846 and degree of OA. The levels of COMP and CS846 significantly increased and a positive correlation was observed between the two biomarkers 10 weeks after OA (26). Another study reported that rheumatoid arthritis patients had significantly higher levels of COMP compared to healthy controls. This verifies the potential of COMP as a marker of articular cartilage injury or cartilage turnover (27).

In the present study, we investigated effects of eight weeks of exercise alone and along with glucosamine supplementation on serum levels of COMP and CS846 in an OA model. Based on the results, exercise training alone or combined with glucosamine supplementation 
reduced cartilage degradation by lowering COMP and CS846 levels. However, the most significant effect was observed in the exercise+glucosamine group compared to the OA group. This indicates that the use of both exercise and glucosamine might have a synergistic protective effect against OA.

Glucosamine is an important precursor of glycoprotein and GAG synthesis. When glucosamine was first proposed for treatment of OA, it was thought to enhance endogenous production of glucosamine and enhance the synthesis of proteoglycan, which is lost early in OA. Oral glucosamine can recover GAG in damaged cartilage, but its mechanism of action is unknown. On the other hand, it has been reported that glucosamine affects the synthesis of type II collagen. In the OA rat models, glucosamine exerts protective effects on cartilage by preserving proteoglycan, inhibition of collagen type II degradation and enhancing collagen type II synthesis in articular cartilage. In addition, glucosamine has a positive effect on bone remodeling, structure and mineralization in the early stages of experimental OA. Exogenous glucosamine has been reported to increase hyaluronic acid production in synovial tissue. This may also be useful for preventing OA development or progression (5). There have been several studies that have suggested the role of glucosamine administration in reducing joint pain in patients with knee OA $(28,29)$. One of the causes of pain in OA is synovium and articular capsule inflammation. Since glucosamine is also an anti-inflammatory drug, it prevents pain by inhibiting inflammation. Small fractures in the subcutaneous bone in OA can cause joint pain. Articular cartilage destruction in any part of the knee joint can cause elongation and spasm with uneven distribution of force on the ligaments, muscles and skin, leading to knee pain. Glucosamine administration, with its role in cartilage production, chondrocyte proliferation and collagen production, avoids pain and prevents unequal distribution of force across various articular segments. Osteophyte production is often caused by disproportionate force on the subcartilage, and osteophytes stretch the nerve terminals below the periosteum and causes pain. Therefore, osteophytes can be prevented by administration of glucosamine and cartilage repair and eliminate one of the mechanisms of pain (30). In a study on horses with OA, Koenig et al. reported that treatment with pentosan polysulfate sodium, N-acetyl glucosamine and sodium hyaluronan increased epitope 846 and GAG concentrations (28). Andersson et al. reported that the effect of physical activity on COMP level was transient (31). In contrast, Kersting et al. reported that muscle activation was the most important mechanical parameter for COMP cartilage changes (32). Mündermann et al. studied serum COMP concentration changes following articular pressure by 30 minutes of walking exercise in patients with knee OA. They reported that serum COMP concentration increased significantly immediately after the training but decreased in the patients after 5.5 hours of exercise (33). According to Roberts (2017), the intensity and type of exercise training can alter the COMP response to exercise-induced loading (34). The role of exercise training in functional adaptation of cartilage tissue suggests the need for further investigation.

\section{CONCLUSION}

Overall, the findings of the present study show that OA leads to an increase in serum COMP and CS846 levels. The combined therapeutic effect of glucosamine combined with exercise to improve knee $\mathrm{OA}$ in rats is more effective than exercise and glucosamine alone. However, it may be more useful to examine the histological findings along with the measured biomarkers for a more accurate assessment of joint damage in OA.

\section{ACKNOWLEDGEMENTS}

We would like to thank the staff of the exercise physiology center of Islamic Azad University of Sari.

\section{CONFLICT OF INTEREST}

All authors declare that there is no conflict of interest. 


\section{REFERENCES}

1. Das SK, Farooqi A. Osteoarthritis. Best Pract Res Clin Rheumatol. 2008; 22: 657-675. [DOI:10.1016/j.berh.2008.07.002] [PubMed] [Google Scholar]

2. Davatchi F, Jamshidi AR, Banihashemi AT, Gholami $\mathrm{J}$, Forouzanfar $\mathrm{MH}$, Akhlaghi $\mathrm{M}$, et al. WHO-ILAR COPCORD study (stage 1, urban study) in Iran. The Journal of rheumatology. 2008; 35(7): 1384-90. [PubMed] [Google Scholar]

3. Zhang W, Moskowitz RW, Nuki G, Abramson S, Altman RD, Arden N, et al. OARSI recommendations for the management of hip and knee osteoarthritis, Part II: OARSI evidence-based, expert consensus guidelines. Osteoarthritis and cartilage. 2008; 16(2): 137-62. [DOI:10.1016/j.joca.2007.12.013] [PubMed] [Google Scholar]

4. Zhang W, Nuki G, Moskowitz RW, Abramson S, Altman RD, Arden NK, et al. OARSI recommendations for the management of hip and knee osteoarthritis: part III: Changes in evidence following systematic cumulative update of research published through January 2009. Osteoarthritis and cartilage. 2010; 18(4): 476-99. [DOI:10.1016/j.joca.2010.01.013] [PubMed] [Google Scholar]

5. Ohnishi A, Osaki T, Matahira Y, Tsuka T, Imagawa T, Okamoto Y, Minami S. Evaluation of the chondroprotective effects of glucosamine and fish collagen peptide on a rabbit ACLT model using serum biomarkers. Journal of Veterinary Medical Science. 2012; 75(4): 12. [PubMed] [Google Scholar]

6. Bay-Jensen AC, Sondergaard BC, Christiansen C, Karsdal MA, Madsen SH, Qvist P. Biochemical markers of joint tissue turnover. Assay and drug development technologies. $\quad 2010 ; \quad 8(1): \quad 118-24$. [DOI:10.1089/adt.2009.0199] [PubMed] [Google Scholar]

7. Petersen SG, Saxne T, Heinegard D, Hansen M, Holm $\mathrm{L}$, Koskinen S, et al. Glucosamine but not ibuprofen alters cartilage turnover in osteoarthritis patients in response to physical training. Osteoarthritis and Cartilage. $\quad 2010 ; \quad$ 18(1): 34-40. [DOI:10.1016/j.joca.2009.07.004] [PubMed] [Google Scholar]

8. Lamprecht ED, Williams CA. Biomarkers of antioxidant status, inflammation, and cartilage metabolism are affected by acute intense exercise but not superoxide dismutase supplementation in horses. Oxidative medicine and cellular longevity. $2012 ; 2012$. [DOI:10.1155/2012/920932] [PubMed] [Google Scholar] 9. Bhatia D, Bejarano T, Novo M. Current interventions in the management of knee osteoarthritis. J Pharm Bioallied Sci. 2013; 5: 30-38. [DOI:10.4103/09757406.106561] [PubMed] [Google Scholar]

10. Francois R. Non-pharmacological approches for the treatment of osteoarthritis. Best Pract Res Clin Rheumatol. 2010; 24: 93-106. [DOI:10.1016/j.berh.2009.08.013] [PubMed] [Google Scholar]

11. Dahmer S, Schiller RM. Glucosamine (Review). Am Fam Physician. 2008; 78: 471-476.
12. Bruyere O, Reginster JY. Glucosamine and chondroitin sulfate as therapeutic agents for knee and hip osteoarthritis (Review). Drugs Aging. 2007; 24: 573580. [DOI:10.2165/00002512-200724070-00005] [PubMed] [Google Scholar]

13. Reginster JY, Bruyere O, Fraikin G, Henrotin Y. Current concepts in the therapeutic management of osteoarthritis with glucosamine (Review). Bull Hosp Jt Dis. 2005; 63: 31-36. [PubMed] [Google Scholar]

14. Herrero-Beaumont G, Ivorra JAR, Trabado MC, Blanco FJ, et al. Glucosamine sulfate in the treatment of knee osteoarthritis symptoms A randomized, doubleblind, placebo-controlled study using acetaminophen as a side comparator. Arthritis Rheum. 2007; 56: 555-567. [DOI:10.1002/art.22371] [PubMed] [Google Scholar]

15. Towheed T, Maxwell L, Anastassiades TP, Shea B, et al. Glucosamine therapy for treating osteoarthritis (Review). Cochrane Database Syst Rev. 2005; 18: CD002946. [DOI:10.1002/14651858.CD002946.pub2] [PubMed] [Google Scholar]

16. Mohsin M. Effects of glucosamine sulfate on primary knee osteoarthritis. Al Ameen J Med Sci. 2008; 1: 42-49. [PubMed] [Google Scholar]

17. Hammad YH, Magid HR, Sobhy MM. Clinical and biochemical study of the comparative efficacy of topical versus oral glucosamine/chondroitin sulfate on osteoarthritis of the knee. Egy Rheumatol. 2015; 37: 8591. [DOI:10.1016/j.ejr.2014.06.007] [PubMed] [Google Scholar]

18. Androeli E, Carpenter CJ, Griggs RC, Bejamin IJ. Cecil essential of medicine. 7th ed. Philadelphia: Saunders. 2007; 845-847. [Google Scholar]

19. Blagojevic M, Jinks C, Jeffery A, Jordan 1. Risk factors for onset of osteoarthritis of the knee in older adults: a systematic review and meta-analysis. Osteoarthritis and cartilage. 2010; 18(1): 24-33. [DOI:10.1016/j.joca.2009.08.010] [PubMed] [Google Scholar]

20. Cifuentes DJ, Rocha LG, Silva LA, Brito AC, RueffBarroso CR, Porto LC, Pinho RA. Decrease in oxidative stress and histological changes induced by physical exercise calibrated in rats with osteoarthritis induced by monosodium iodoacetate. Osteoarthritis and Cartilage. 2010; 18(8): 1088-95. [DOI:10.1016/j.joca.2010.04.004] [PubMed] [Google Scholar]

21. Galois L, Etienne S, Grossin L, Watrin-Pinzano A, Cournil-Henrionnet C, Loeuille D, et al. Dose-response relationship for exercise on severity of experimental osteoarthritis in rats: a pilot study. Osteoarthritis and $\begin{array}{llll}\text { cartilage. } & 2004 ; & \text { 12(10): }\end{array}$ [DOI:10.1016/j.joca.2004.06.008] [PubMed] [Google Scholar]

22. Gupta S, Hawker GA, Laporte A, Croxford R, Coyte PC. The economic burden of disabling hip and knee osteoarthritis (OA) from the perspective of individuals living with this condition. Rheumatology (Oxford). 2005; 44(12): 1531-7. [DOI:10.1093/rheumatology/kei049] [PubMed] [Google Scholar]

23. Malfait AM, Little CB. On the predictive utility of animal models of osteoarthritis. Arthritis research \& therapy. 2015; 17(1): 225. [DOI:10.1186/s13075-0150747-6] [PubMed] [Google Scholar] 
24. Wen ZH, Tang CC, Chang YC, Huang SY, Hsieh SP, Lee $\mathrm{CH}$, et al. Glucosamine sulfate reduces experimental osteoarthritis and nociception in rats: association with changes of mitogen-activated protein kinase in chondrocytes. Osteoarthritis and Cartilage. 2010; 18(9): 1192-202. [DOI:10.1016/j.joca.2010.05.012] [PubMed] [Google Scholar]

25. Ren G, Krawetz RJ. Biochemical Markers for the Early Identification of Osteoarthritis: Systematic Review and Meta-Analysis. Molecular diagnosis \& therapy. 2018; 22(6): 671-82. [DOI:10.1007/s40291-018-0362-8] [PubMed] [Google Scholar]

26. Ma T, Zhang Z, Song X, Bai H, Li Y, Li X, Zhao J, Ma Y, Gao L. Combined detection of COMP and CS846 biomarkers in experimental rat osteoarthritis: a potential approach for assessment and diagnosis of osteoarthritis. Journal of orthopaedic surgery and research. 2018; 13(1): 230. [DOI:10.1186/s13018-018-0938-3] [PubMed] [Google Scholar]

27. Al-Dalaen S, Al-Qtaitat A, Al-Rawashdeh M, Alzyoud J, Al-Maathadi A. Rheumatoid arthritis: hyaluronic acid and cartilage oligomeric matrix protein as predictors of the disease progression. Biomedical and Pharmacology Journal. 2016; 9(1): 15-23. [DOI:10.13005/bpj/903] [PubMed] [Google Scholar]

28. Koenig TJ, Dart AJ, McIlwraith CW, Horadagoda N, Bell RJ, Perkins N, et al. Treatment of Experimentally Induced Osteoarthritis in Horses Using an Intravenous Combination of Sodium Pentosan Polysulfate, N-Acetyl Glucosamine, and Sodium Hyaluronan. Veterinary surgery. 2014; 43(5): 612-22. [DOI:10.1111/j.1532950X.2014.12203.x] [PubMed] [Google Scholar]

29. Naito K, Watari $T$, Furuhata A, Yomogida S, Sakamoto K, Kurosawa $\mathrm{H}$, et al. Evaluation of the effect of glucosamine on an experimental rat osteoarthritis model. Life sciences. 2010; 86(13-14): 538-43. [DOI:10.1016/j.1fs.2010.02.015] [PubMed] [Google Scholar]
30. Arti HR, Azemi ME. Comparing the effect of glucosamine and glucosamine with alendronate in symptomatic relieve of degenerative knee joint disease: a double-blind randomized clinical trial study. Jundishapur journal of natural pharmaceutical products. 2012; 7(3): 87. [DOI:10.5812/jjnpp.3405] [PubMed] [Google Scholar]

31. Andersson ML, Thorstensson CA, Roos EM, Petersson IF, Heinegård D, Saxne T. Serum levels of cartilage oligomeric matrix protein (COMP) increase temporarily after physical exercise in patients with knee osteoarthritis. BMC Musculoskeletal disorders. 2006; 7(1): 98. [DOI:10.1186/1471-2474-7-98] [PubMed] [Google Scholar]

32. Kersting UG, Stubendorff JJ, Schmidt MC, Brüggemann GP. Changes in knee cartilage volume and serum COMP concentration after running exercise. Osteoarthritis and cartilage. 2005; 13(10): 925-34. [DOI:10.1016/j.joca.2005.06.005] [PubMed] [Google Scholar]

33. Mündermann A, King KB, Smith RL, Andriacchi TP. Change in serum COMP concentration due to ambulatory load is not related to knee OA status. Journal of Orthopaedic Research. 2009; 27(11): 1408-13. [DOI:10.1002/jor.20908] [Google Scholar]

34. Roberts HM. The Effect of Exercise and Ageing on Morphology and Biomarkers of Knee Articular Cartilage in Healthy Humans. Bangor University (United Kingdom); 2017. [Google Scholar]

How to Cite:

Biniaz B, Abbaszadeh H, Farzanegi P, Hejazi M[Effect of Glucosamine Sulfate Alone and Combined with Moderate Intensity Exercise on Serum Levels of CS 846 Epitope and Cartilage Oligomeric Matrix Protein in a Rat Model of Osteoarthritis]. mljgoums. 2021: 15(2): 35-41 DOI: $10.29252 / \mathrm{mli} .15 .2 .35$

(C) The authors 